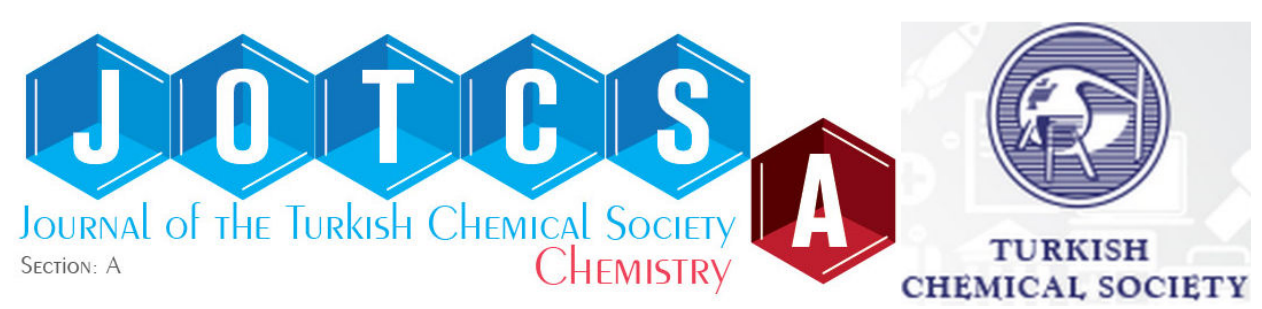

\title{
A Versatile Water-Soluble Ball-Type Phthalocyanine as Potential Antiproliferative Drug: The Interaction with G- Quadruplex Formed From Tel 21 and cMYC
}

\author{
Efkan BAĞDA ${ }^{1}$, Esra BAĞDA², Ebru YABAŞ ${ }^{3 *}$ \\ ${ }^{1}$ Department of Molecular Biology and Genetics, Cumhuriyet University Faculty of \\ Science, 58140, Sivas, Turkey. \\ ${ }^{2}$ Department of Analytical Chemistry, Cumhuriyet University Faculty of Pharmacy, \\ 58140, Sivas, Turkey. \\ ${ }^{3}$ Department of Chemistry and Chemical Processing Technologies, Cumhuriyet \\ University Imranlı Vocational School, 58980, Sivas, Turkey.
}

\begin{abstract}
G-quadruplexes are biologically important DNA conformations exist generally in guanine-rich segments of DNA, such as telomere and proto-oncogene. The formation of these secondary structures is thought to inhibit the expression of certain genes, such as the inhibition of telomerase. The inhibition of telomerase and suppression of a specific gene expression are important approaches for interruption of cancer cell's proliferation. In the present study, the effect of a versatile water soluble ball-type phthalocyanine (BtPc) on G-quadruplex formation and stabilization was investigated to demonstrate its potential usage in cancer chemotherapy. Two important guanine rich oligomers, CMYC and Tel 21 were used as G-quadruplex former sequence. To the best of our knowledge, this is the first study about the interaction of a BtPc with G-quadruplex structures. The interactions of the compound with $\mathrm{G}$-quadruplex molecules were monitored spectrophotometrically. The binding constants were calculated from BenesiHildebrand equation and the highest binding constant $\left(0.1114 \mu \mathrm{M}^{-1}\right)$ was found for Tel 21 in the presence of $\mathrm{KCl}$. The structural differentiations of G-quadruplex after binding were investigated with circular dichroism spectrophotometry. The CD spectra were demonstrated the stabilization of both parallel and anti-parallel Tel $21 \mathrm{G}$-quadruplex in the presence of $\mathrm{KCl}$ and stabilization of anti-parallel form in the absence of $\mathrm{KCl}$. The stability of the parallel structure was achieved for CMYC in the absence of $\mathrm{KCl}$ up to $4.10 \mu \mathrm{M}$ of Pc. The disruption of staking of parallel form was achieved for CMYC in the presence of $\mathrm{KCl}$. The replacement ability of the molecule with a known DNA binding molecule, ethidium bromide, was clarified fluorometrically. The Stern-Volmer studies were conducted for determination of the quenching mechanism. The strong interaction of the molecules (BtPc with oligomer) showed us the potential usage of these drug conjugates for targeted photodynamic therapy in the future.
\end{abstract}

Keywords: G-quadruplex, CD spectroscopic titration, telomere, oncogene.

Cite this: 1. Bağda E, Bağda E, Yabaş $E$. A versatile water soluble ball-type phthalocyanine as potential antiproliferative drug: the interaction with G-quadruplex formed from Tel 21 and CMYC. JOTCSA. 2017;4(2):103-20.

Submitted: January 27, 2017. Revised: March 23, 2017. Accepted: April 25, 2017.

DOI: $10.18596 /$ jotcsa. 288284

*Corresponding author. E-mail: yabasebru@gmail.com, eyabas@cumhuriyet.edu.tr 


\section{INTRODUCTION}

Guanine-rich segments of DNA can fold into four stranded structures which are named G-quadruplexes [1-3]. The formation of G-quadruplexes was known for more than 40 years, but their existence in vivo has been discovered recently $[4,5]$. According to Bhattacharjee et al., DNA can fold into a G-quadruplex structure, formed by $\Pi \rightarrow \Pi$ stacking of G-quartets [4]. G-quartets are composed of cyclic arrangement of four guanine molecules connected via Hoogsteen hydrogen bonds and they are stabilized by a central monovalent cation, such as $\mathrm{K}^{+}$or $\mathrm{Na}^{+}[4]$.

G-quadruplexes can exist in telomeric and promotor region of oncogenes $[6,7]$. The presence of these highly ordered structures in telomeric end of DNA and oncogene regulatory regions are evident $[7,8]$ and they have biologically important roles. The guanine rich DNA sequences exist in different sequence of chromosome $[9,10]$. The telomeric region of DNA is one of most known sequence of them. According to Gao et al. [11], telomerase is responsible for adding telomere repeats to telomeric DNA to maintain the integrity and stability of chromosome.

In normal somatic cells, the length of telomere sequence shortens at every cell division until they enter replicative senescence [12]. The telomerase activities in normal cell are highly lower compared to cancer cells, so the inhibition of telomerase is an important target for cancer chemotherapy. According to Gao et al., it is very important that, telomerase binds to linear telomere DNA, not folded, like quadruplex structure [11]. The recognization of the structure by the enzyme cannot be achieved when the sequence forms secondary structures like G-quadruplexes. So, the formation of G-quadruplex structures results in inhibition of telomerase and interruption of replication of DNA [13]. Considering this point, the effective Gquadruplex stabilizing agents are promising alternative in cancer therapeutics. Besides, G-quadruplexes are thought to be useful drug delivery vehicles for especially cancer therapy [14]. Their thermal stability and easy/ low cost production make them alternative targetable drug carriers. The delivering the drug to a target cell or tissue via conjugation with a specific G-quadruplex structure seems highly feasible. So, the binding a potential drug with a suitable G-quadruplex structure is important for its potential therapeutic usage. 
Phthalocyanines are attractive molecules due to their interesting biological applications [15]. With differentiation of the peripheral side groups and central metal ions, various different types of new phthalocyanines can be synthesized. The important biological characteristics of phthalocyanines such as antibacterial, antifungal, antioxidant activity made scientists focus on the applicability of these molecules in different medical areas.

The interesting studies related with the interaction of G-quadruplexes with different phthalocyanines have been reported in the literature. Among them, generally mono type phthalocyanines were employed. Synthesis of different types of phthalocyanines (double-decker, clamshell, etc.) have been also accomplished by different research groups [16]. The first studies about synthesis of BtPc were conducted by Tolbin et al $[17,18]$ but biological applications of BtPcs are rare in the literature.

\section{Literature survey}

The G-quadruplex stabilization effect of special molecules has attracted attention due to their potential usages in cancer therapy. There are interesting examples of Gquadruplex interactive molecules in the literature: Bhattacharjee et al. found that Zn(II) 5,10,15,20-tetrakis(N-methyl-4-pyridyl)porphyrin was a G-quadruplex interactive molecule [4]. They showed unambiguously, and for the first time, that ZnTMPyP4 greatly facilitated the folding of d(TAGGG)2 into parallel G-quadruplex, probably by driving the equilibrium between unfolded and folded G-quadruplex toward the latter under otherwise unfavorable conditions (low amount of potassium ions). Chan et al. studied with methylene blue derivatives as a promising scaffolds for binding the CMYC oncogene G-quadruplex DNA [6]. They used a structure-based lead optimization approach to generate MB derivatives that displayed superior binding affinity and selectivity for the CMYC G-quadruplex over double-stranded DNA or other G-quadruplex structures. Gao et al. worked with 2,7-diamino-10-(3,5dimethoxy)benzyl-9(10H)-acridone derivatives as potent telomeric G-quadruplex DNA ligands [11]. They showed that some of the compounds displayed good antiproliferative activity against leukemia CCRF-CEM cells, among which compound $6 a$ containing dimethylamine substituents at the terminal $\mathrm{C} 2$ and $\mathrm{C} 7$ positions exhibited the highest cytotoxicity with $\mathrm{IC}_{50}$ at $0.3 \mu \mathrm{M}$. In addition, compound $6 \mathrm{a}$ showed little toxicity against normal 293T cells proliferation with IC50 more than 100 $\mu \mathrm{M}$. The study conducted by Hassani et al. was about the interaction of copper porphyrazines and phthalocyanines with human telomeric G-quadruplex DNA. They found that interaction of the cationic porphyrazines was remarkably stronger than 
the anionic phthalocyanines [25]. Another study concerning the interaction of thymoquinone with G-quadruplex DNA was conducted by Salem et al. [26]. They concluded that interaction of thymoquinone with G-quadruplex will contribute to the inhibition of telomerase enzyme and cancer's proliferation. Yaku et al. studied the telomere G-quadruplex-binding and telomerase-inhibiting capacity of two cationic and two anionic G-quadruplex ligands [27]. They concluded that ligands that bind to G-quadruplex via interactions such $\Pi-\Pi$ stacking and hydrogen bonding may represent superior telomerase inhibitors in living cell media.

Phthalocyanines are highly interesting molecules and different types of phthalocyanines (mono, ball-type, double-decker, etc.) and similar molecules have been synthesized due to their usages in medicinal area. In the present study, water soluble BtPc molecule was employed as G-quadruplex interactive molecule.

In our previous study, we have synthesized a new water-soluble phthalocyanine, BtPc, and reported the ct- DNA binding ability [15]. As mentioned in many study existing in the literature [19-21], G-quadruplexes are important therapeutic targets. In the present study, in order to clarify the usage of synthesized compound in cancer therapy, the interaction of BtPc molecule with G-quadruplex former oligonucleotides (Tel 21 and CMYC) were investigated in detail. The interactions were clarified with ultraviolet visible (UV-Vis.) and circular dichroism (CD) spectrophotometric methods. An ethidium bromide ( $\mathrm{EtBr}$ ) replacement assay was conducted to understand the interaction closely. The experiments were done in the presence and absence of potassium chloride $(\mathrm{KCl})$ because monovalent cations are known as G-quadruplex stabilization agents [22]. In the first part of our experiments, with the presence of $\mathrm{KCl}$, the interaction of BtPc with pre-formed G-quadruplex was studied. In the absence of $\mathrm{KCl}$, the G-quadruplex forming or stabilizing ability of BtPc was investigated.

\section{EXPERIMENTAL}

\section{Reagents and instruments}

All chemicals were of analytical and molecular biology grade. The oligomers (CMYC and Tel 21) were purchased from Alpha DNA and used without further purification. The sequence of cMYC was TGAGGGTGGGAGGGTGGGAA and Tel 21 was GgGTtAGGGTTAGGGTTAGGGG ( $5^{\prime}$ to $\left.3^{\prime}\right)$. The stock $(100 \mu \mathrm{M})$ oligomer solutions 
were prepared in $\mathrm{pH} 7.4 \mathrm{TE}$ (Tris-EDTA, $10 \mathrm{mM}$ Tris $\mathrm{HCl}, 1 \mathrm{mM}$ EDTA) buffer in the presence and absence of $150 \mathrm{mM} \mathrm{KCl}$. After dissolution of oligomers, the solutions were annealed at $95{ }^{\circ} \mathrm{C}$ for five minutes and cooled to room temperature slowly. The solutions were incubated at $+4{ }^{\circ} \mathrm{C}$ at least overnight. The double distilled water (after autoclaving) was used all throughout the experiments. The BtPc solution was prepared in both salty $(150 \mathrm{mM} \mathrm{KCl})$ and salt-free $\mathrm{pH} 7.4 \mathrm{TE}$ buffer. BtPc solution was magnetically stirred at least four hours for complete dissolution.

All spectrophotometric measurements were conducted with a MAPADA series 6spectrophotometer with using a $1 \mathrm{~cm}$ pathway quartz cell. A Shimadzu RF 5301 fluorescence spectrophotometer was used for spectrofluorometric measurements. $\mathrm{pH}$ measurements were conducted with Sartorius basic $\mathrm{pH}$ meter with a glass electrode. The $\mathrm{pH}$ meter was calibrated with using standard buffer solutions $(\mathrm{pH}=4.0,7.0,10)$ purchased from Reagecon (Sahnnon Free zone, Ireland). A Shimadzu analytical balance was used for weighing the chemicals. CD spectra were collected with a Jasco J-815 CD spectrophotometer.

\section{Synthesis of BtPc}

The detailed synthesis route was given in our previous study [15]. The synthesis steps were as follows: BtPc was synthesized by the cyclotetramerization of synthesized triethanolamine-substituted phthalonitrile derivative and water solubility of the compound was achieved after quaternization.

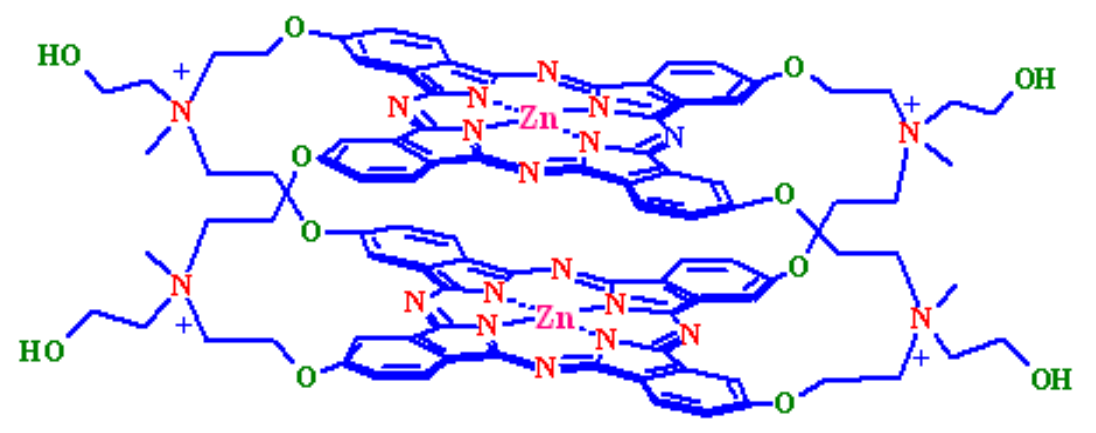

Water Soluble Ball Type Phthalocyanine.

\section{Analysis by UV-Vis. and CD Spectroscopy}

UV-Vis spectrophotometric experiments were performed with gradual addition of aliquots of Tel 21 and CMYC to BtPc solution (final oligomer concentrations were between 3.23-8.54 $\mu \mathrm{M}$ ). The experiments were conducted with and without $150 \mathrm{mM}$ 
$\mathrm{KCl}$. The absorbance values were recorded against buffer solution with or without $\mathrm{KCl}$. In order to evaluate the binding constant, spectrophotometric data were further processed with the Benesi-Hildebrand equation given below $[8,23]$ :

$$
\frac{1}{\Delta A_{\lambda}}=-\frac{1}{\left(\varepsilon_{b}-\varepsilon_{f}\right)\left[L_{t}\right]}+\frac{1}{\left(\varepsilon_{b}-\varepsilon_{f}\right)\left[L_{t}\right] K_{B H}[D N A]}
$$

Where the $\Delta \mathrm{A}$ is the absorbance chance for corresponding $\lambda_{\max }$ of BtPc ( $\lambda_{\max }$ is 602 $\mathrm{nm}$ in the presence of $\mathrm{KCl}$ and $611 \mathrm{~nm}$ in the absence of $\mathrm{KCl}$ ), $\varepsilon_{b}$ and $\varepsilon_{\mathrm{f}}$ stands for the molar extinction coefficients of bound and free ligand, $\left[\mathrm{L}_{\mathrm{t}}\right]$ is the total concentration of BtPc. The binding constant of DNA to Pc was evaluated with using slope and intercept values of Benesi-Hildebrand plot.

CD spectrophotometric titrations were conducted with gradual addition of BtPc (21 $\mu \mathrm{M})$ to constant concentrations of Tel $21(7.0 \mu \mathrm{M})$ and cMYC $(2.4 \mu \mathrm{M})$ solutions. After addition of Pc solution, the solution was incubated for 5 minutes. The CD spectra were collected with the average of three scans. The spectra were taken against reagent blank.

\section{Determination of quenching effect of BtPc molecule}

Fluorometric experiments were done to evaluate the replacement ability of BtPC molecule with EtBr molecule. For the replacement assay, $5 \mu \mathrm{L}$ of $10 \mathrm{mg} / \mathrm{mL} \mathrm{EtBr}$ was introduced to the oligomeric solution $(33 \mu \mathrm{M})$. After incubation for ten minutes, predetermined aliquots of BtPc $(21 \mu \mathrm{M})$ solution was added to the mixture and the fluorometric intensity was monitored. The quenching effect of BtPc molecule was determined via Stern-Volmer equation given below [24]:

$$
\frac{F_{0}}{F}=1+K_{S V}[B t P c]
$$

Where $\mathrm{F}_{0}$ was the fluorescence intensity of EtBr-oligomer complex and $\mathrm{F}$ was the fluorescence intensity of EtBr-oligomer complex after addition of BtPc. [BtPc] was the concentration of the BtPc, and $\mathrm{K}_{\mathrm{sv}}$ was the Stern-Volmer constant which could be used for the deduction of the binding/replacement ability of BtPc molecule. The slope of $\mathrm{F}_{0} / \mathrm{F}$ versus $[\mathrm{BtPc}]$ plot gave the $\mathrm{K}_{\mathrm{sv}}$ value. 


\section{RESULTS AND DISCUSSION}

\section{Evaluation of UV-Vis. spectral data}

UV-Vis spectrophotometry is the simplest, economic and commonly used method for studying both the stability of DNA secondary structures like G-quadruplexes and their interactions with different ligands [28].

As stated in our previous study [15] the UV-Vis. spectrum of BtPc molecule showing strong absorption band between $600-700 \mathrm{~nm}$. In the present study, the absorption spectra of BtPc in $\mathrm{pH} 7.4$ buffer with and without $\mathrm{KCl}$ were recorded. The presence of $\mathrm{KCl}$ resulted in hypochromism along with blue shift $(9 \mathrm{~nm})$ in Q-band region. These results represented the formation of aggregates in the presence of strong electrolyte [24].

The spectrophotometric analysis gives some information about the binding of two molecules. In generally, ligands show an absorption band in visible region and the interaction can be easily examined by the shifting of the $\lambda_{\max }$ [29]. For DNA - small molecule interaction, the differentiation in absorbance and wavelength shift are useful data for prediction of strength of the interaction. The strong interactions generally result in significant differentiation in absorbance (hyper- or hypochromism) and sometimes in wavelength (bathochromic or hypsochromic shifts).

In the present study, as can be seen from Figure 1, the absorbance value of BtPc molecule has fallen from about 1.56 to 1.05 when the concentration of cMYC increased from 0-6.25 $\mu \mathrm{M}$ and a red shift about $21 \mathrm{~nm}$ occurred in the absence of $\mathrm{KCl}$. On the other hand, for the same oncogene and for the same concentrations, the absorbance value of the BtPc molecule decreased from about 1.26 to 0.90 (for 0$6.25 \mu \mathrm{M})$ in the presence of $\mathrm{KCl}$. The wavelength shift was also $21 \mathrm{~nm}$. 


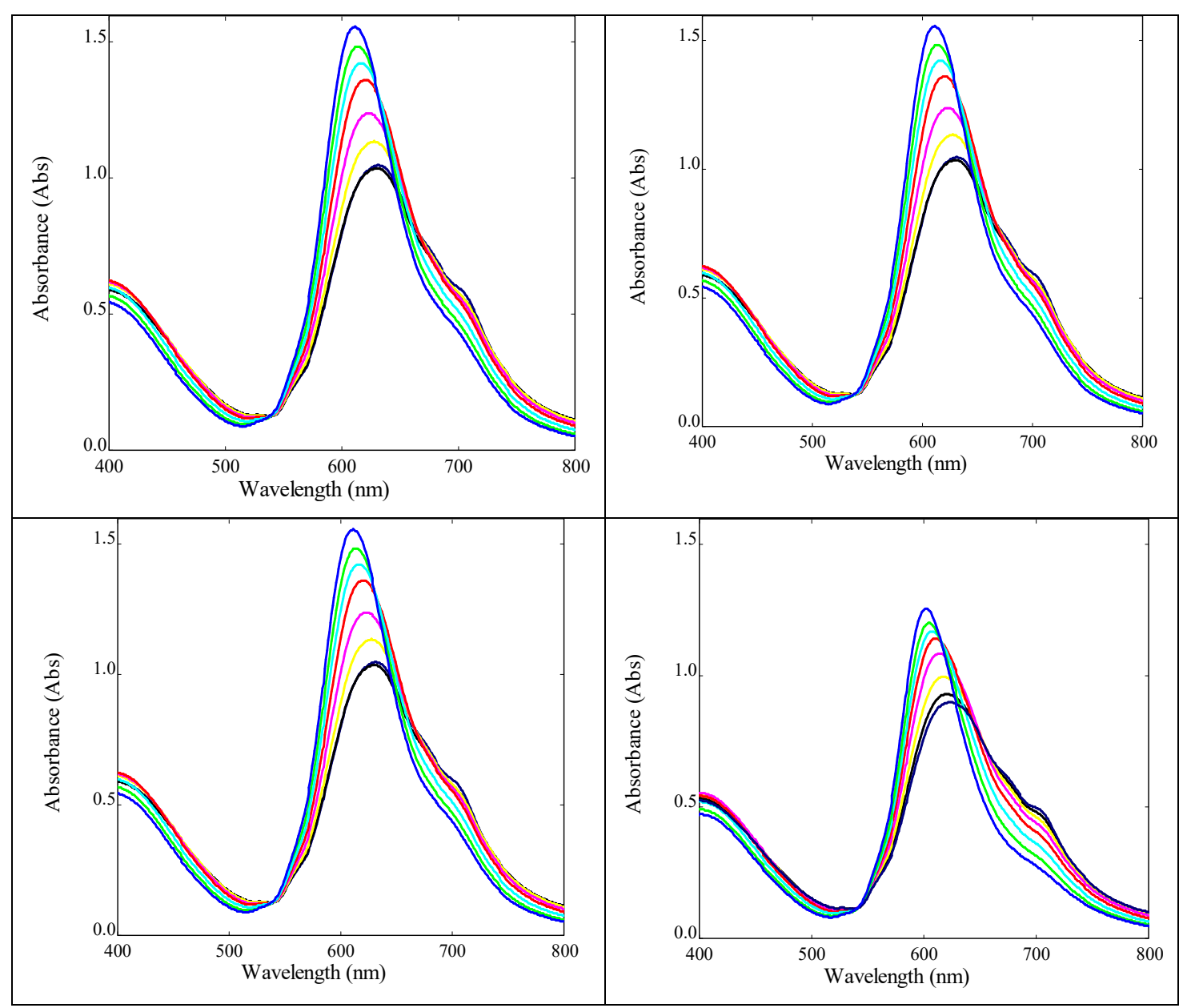

Figure 1: Change in molecular absorbance spectra of BtPc in $Q$ band region with addition of cMYC in the absence (upper left) and presence (upper right) of $150 \mathrm{mM}$ $\mathrm{KCl}$, with addition of Tel 21 in the absence (lower left) and presence (lower right) of $150 \mathrm{mM} \mathrm{KCl}$.

In the spectrophotometric titration of BtPc with Tel 21, the concentration of BtPc and Tel 21 was adjusted the same values of CMYC (0-6.25 $\mu \mathrm{M})$. In the titration of BtPC with Tel 21, the absorbance value fallen from 1.56 to 1.16 with a red shift of $22 \mathrm{~nm}$ in the $\mathrm{KCl}$ free medium. The absorbance decreased from 1.28 to 1.02 accompanied with a red shift of $15 \mathrm{~nm}$ in the presence of $\mathrm{KCl}$. The absorbance decreases were the obvious indicators of the BtPc oncogene/G-quadruplex interactions. The spectral data were treated with Benesi-Hildebrand equation. As shown in Table 1, the binding constant of Tel 21 had the highest value (about $0.1114 \mu^{-1}$ ) in the presence of $\mathrm{KCl}$ (as can be seen in section CD spectroscopic analysis, the elipticity values of Tel 21 at 265 and $290 \mathrm{~nm}$ increased in the presence of $\mathrm{KCl}$, which confirmed stabilization of both parallel and anti-parallel structures with BtPc molecule). 
As can be seen in Table 1, for all spectral analyses, bathochromic shifts were observed for all circumstances. This was due to combination of BtPc's $n$ electrons and $n$ electrons of bases. The energy level of $\Pi-\Pi^{*}$ transition decreases [29].

Table 1: Spectrophotometric titration data and Benisi-Hildebrand binding constants.

\begin{tabular}{llll}
\hline & & K binding constant, $\mu \mathrm{M}^{-1}$ & $\lambda$ shift, $\mathrm{nm}$ \\
\hline \multirow{2}{*}{ Without $\mathrm{KCl}$} & cMYC & $0.0743 \pm 0.0023$ & 21 \\
\multirow{2}{*}{ With KCl } & Tel 21 & $0.0654 \pm 0.0067$ & 22 \\
& CMYC & $0.0718 \pm 0.0042$ & 21 \\
& Tel 21 & $0.1114 \pm 0.010$ & 15 \\
\hline
\end{tabular}

\section{CD spectroscopic analysis}

DNA segments can adopt various structures that are dependent on their environment. $C D$ is a powerful tool for clarifying the secondary structure of biomolecules and binding characteristics of small molecules [29]. The CD spectrum of parallel G-quadruplex generally displays two major peaks with a positive elipticity at about $260 \mathrm{~nm}$ and a negative at about $240 \mathrm{~nm}$. For anti-parallel structure, a positive at about $290 \mathrm{~nm}$ and a negative at about $240 \mathrm{~nm}$ are characteristics of the spectrum [29].

In the present study, as can be seen from Figure 2, a strong positive peak at about $260 \mathrm{~nm}$, and a negative peak around $240 \mathrm{~nm}$ was due to formation of parallel structure of G-quadruplex for CMCY. The CD spectrum of Tel 21 showed a negative band around $240 \mathrm{~nm}$ and, two positive peaks around 260 and $295 \mathrm{~nm}$. The presence of these two characteristic peaks for Tel 21 was an obvious indicator of presence of two types of the structure in the solution. The titration of CMYC with BtPc caused no significant change in $C D$ spectrum, e.g., significant change in wavelength, or appearance or fully disappearance of a band. The decrease in elipticity at $260 \mathrm{~nm}$ during titration of CMYC with BtPc in the presence of $150 \mathrm{mM}$ of $\mathrm{KCl}$ indicated disruption of staking of parallel form of G-quadruplex. [30]. Controversially, in $\mathrm{KCl}$ free medium, an increase in the parallel conformation of CMYC with addition of BtPC was observed. According to Saleem et al. [26], a decrease in CD intensity implies the intercalation type binding, and an increase shows the groove binding. From the results, it was estimated that the binding of BtPc to CMYC was intercalative in the presence of $\mathrm{KCl}$ and groove binding in the absence of $\mathrm{KCl}$. 


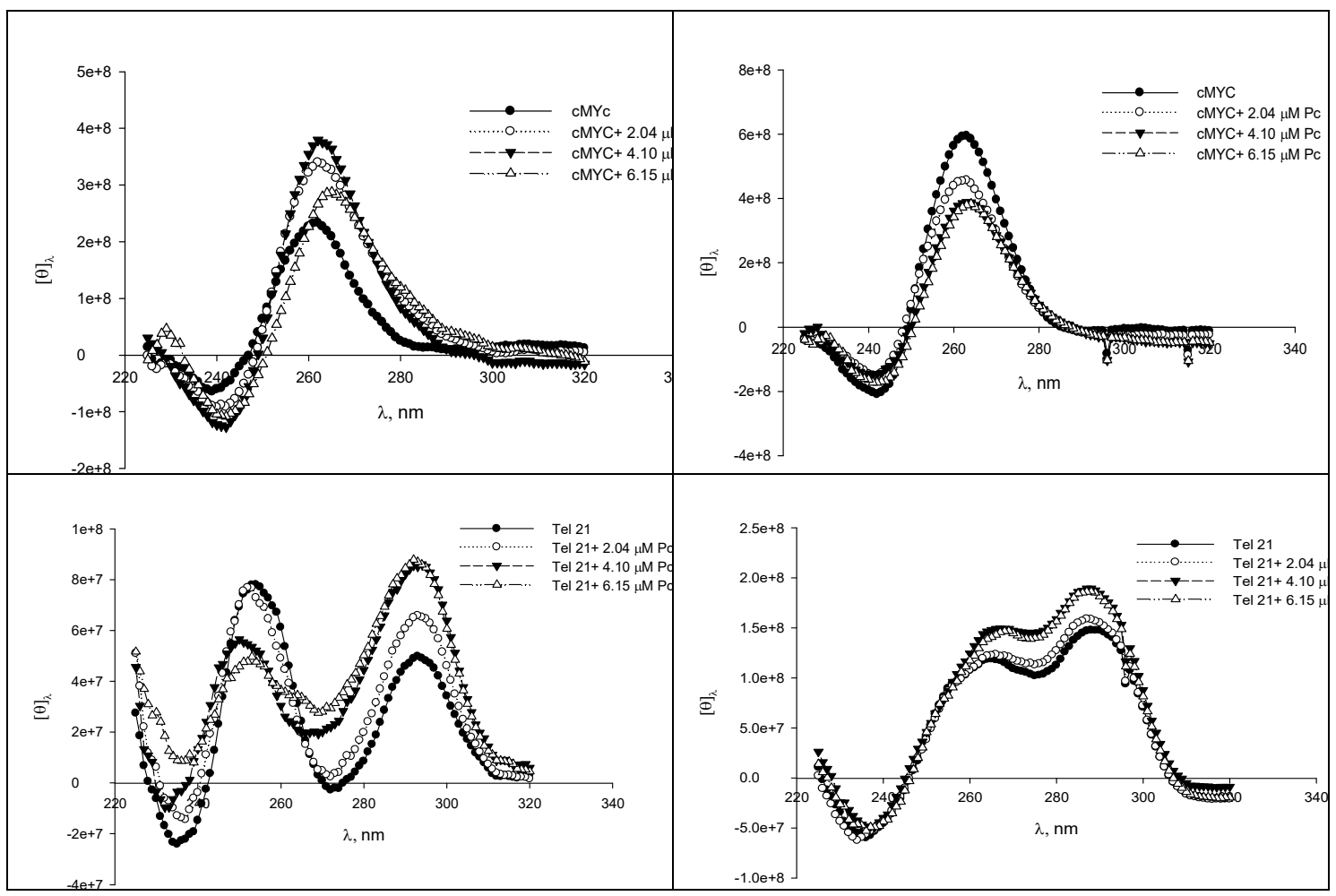

Figure 2: Change in CD spectrum of CMYC with addition of BtPc in the absence (upper left) and presence (upper right) of $150 \mathrm{mM} \mathrm{KCl}$, spectrum of Tel 21 with addition of BtPc in the absence (lower left) and presence (lower right) of $150 \mathrm{mM}$ $\mathrm{KCl}$.

The titration of Tel 21 with BtPc in the presence of $\mathrm{KCl}$ caused the stabilization of hybrid type of structure, e.g. the elipticity of both bands at 260 and $290 \mathrm{~nm}$ increased with increasing the concentration of BtPc. As stated above, an increase in elipticity both at 260 and $290 \mathrm{~nm}$ might be an evidence of binding as groove type in the presence of $\mathrm{KCl}$. In contrast, as shown in Figure 2, in $\mathrm{KCl}$ free medium, when the elipticity of $260 \mathrm{~nm}$ decreased, the elipticity of $290 \mathrm{~nm}$ increased with an increase in concentration of BtPc. These results implied the conversion of parallel structure of Tel 21 to anti-parallel structure favorably with addition of BtPc.

Briefly, addition of BtPc to CMYC resulted in a distortion of parallel structures in the presence of $\mathrm{KCl}$, conversion of irregular oligomers to parallel type G-quadruplex structure in the absence of $\mathrm{KCl}$. Secondly, addition of BtPc to Tel 21 favored the formation of both parallel and anti-parallel structures from irregular form in the presence of $\mathrm{KCl}$ and resulted in conversion to anti-parallel structure from parallel structure in the absence of $\mathrm{KCl}$. As a whole, as can be seen from $\mathrm{CD}$ spectra, binding 
of BtPc molecule to G-quadruplex structure induced structural deviations. As stated by Vivec et al, this type of structural switch has been found for other studies [19].

\section{Fluorescence replacement assay}

$\mathrm{EtBr}$ is a well-known dsDNA intercalator reagent that used for much biological and molecular biological application. It also shows affinity towards G-quadruplex molecules [31,32]. Koeppel et al. showed the affinity of $\mathrm{EtBr}$ derivatives to Gquadruplex structures [32]. Fluorescence intensity of EtBr greatly increases in the presence of G-quadruplex. In the present study the fluorescence intensity of cMYC increased about 80 times and 100 times in the presence and absence of $\mathrm{KCl}$. The fluorescence intensity of Tel 21 also increased in the presence of EtBr. From the point of view, we done replacement assay with $\mathrm{EtBr}$. The fluorescence intensities of Gquadruplex DNA-EtBr system decreased with the addition of quencher molecule, e.g. BtPc molecule (from 0.0 to $5.2 \mu \mathrm{M}$ ) (Figures $3 a$ and $3 b$ ). As shown in Figure 4, SternVolmer plots were deviated from linearity, which implied that the quenching mechanisms were consisting of both static and dynamic quenching. The regression coefficient of Stern-Volmer had the lowest value for CMYC-EtBr replacing with BtPc in the presence of $\mathrm{KCl}\left(r^{2}=0.92\right)$ (Figure 4). The regression coefficient of Stern-Volmer plots for Tel 21 in the presence and absence of $\mathrm{KCl}$ and $\mathrm{CMYC}$ in the absence of $\mathrm{KCl}$ were equal or higher than 0.97 .

For the linear part of Stern-Volmer plot, e.g. for the $5.56 \times 10^{-7}-3.76 \times 10^{-6} \mathrm{M}$ of BtPc of which the regression coefficient higher than 0.98 , the Stern-Volmer constants were calculated (they can be seen in Table 2). In the presence of $\mathrm{KCl}$, $\mathrm{CMYC}$ had the highest $\mathrm{K}_{\mathrm{sv}}$ constant. This might be an evidence of easiness of EtBr replacement. With taking account CD spectrophotometric results, addition of BtPC to CMYC resulted in decrease in parallel structure and increase in perturbation because of intercalation of BtPc. 

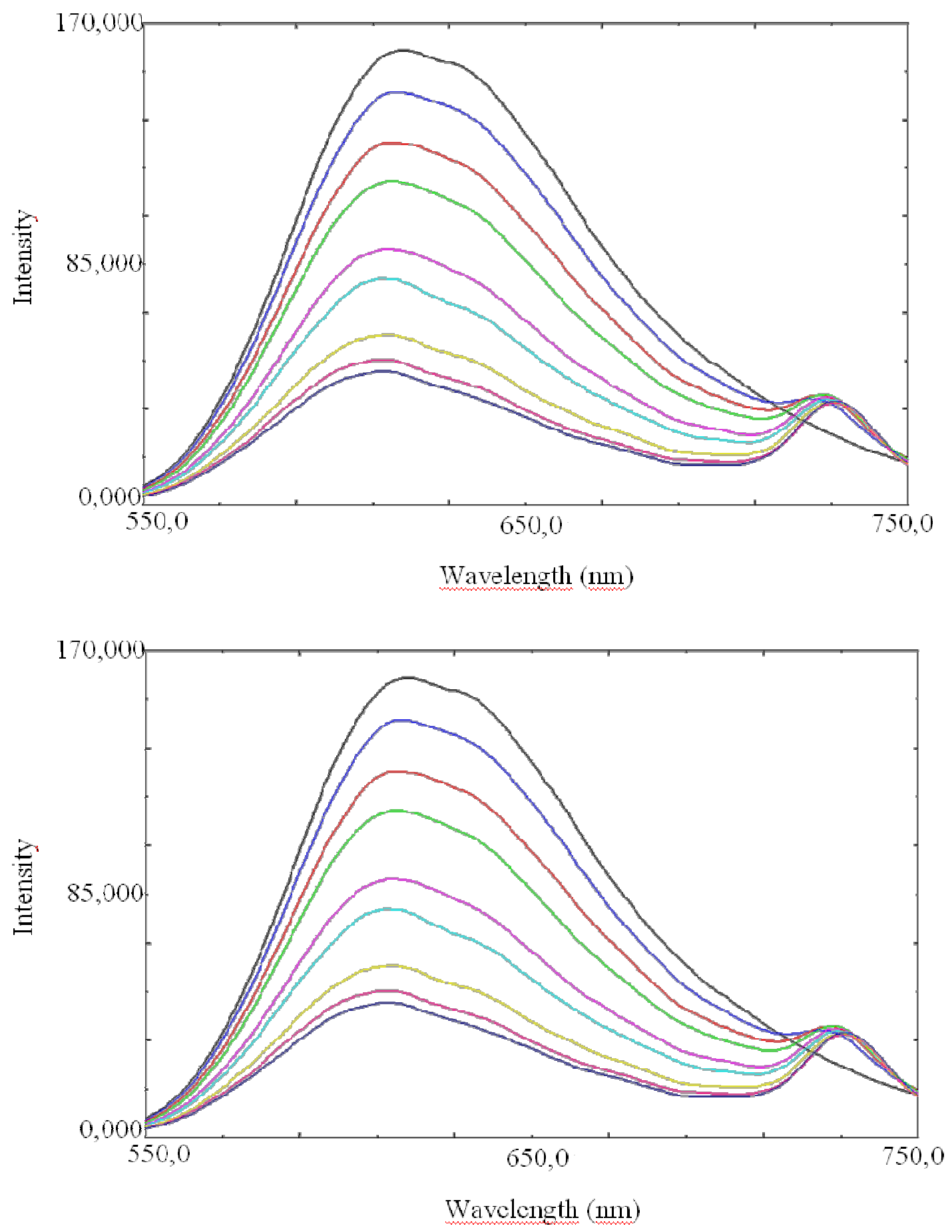

Figure 3a: Change in fluorometric intensity of EtBr- CMYC with addition of BtPc in the absence (up) and presence (down) of $150 \mathrm{mM} \mathrm{KCl}$. 


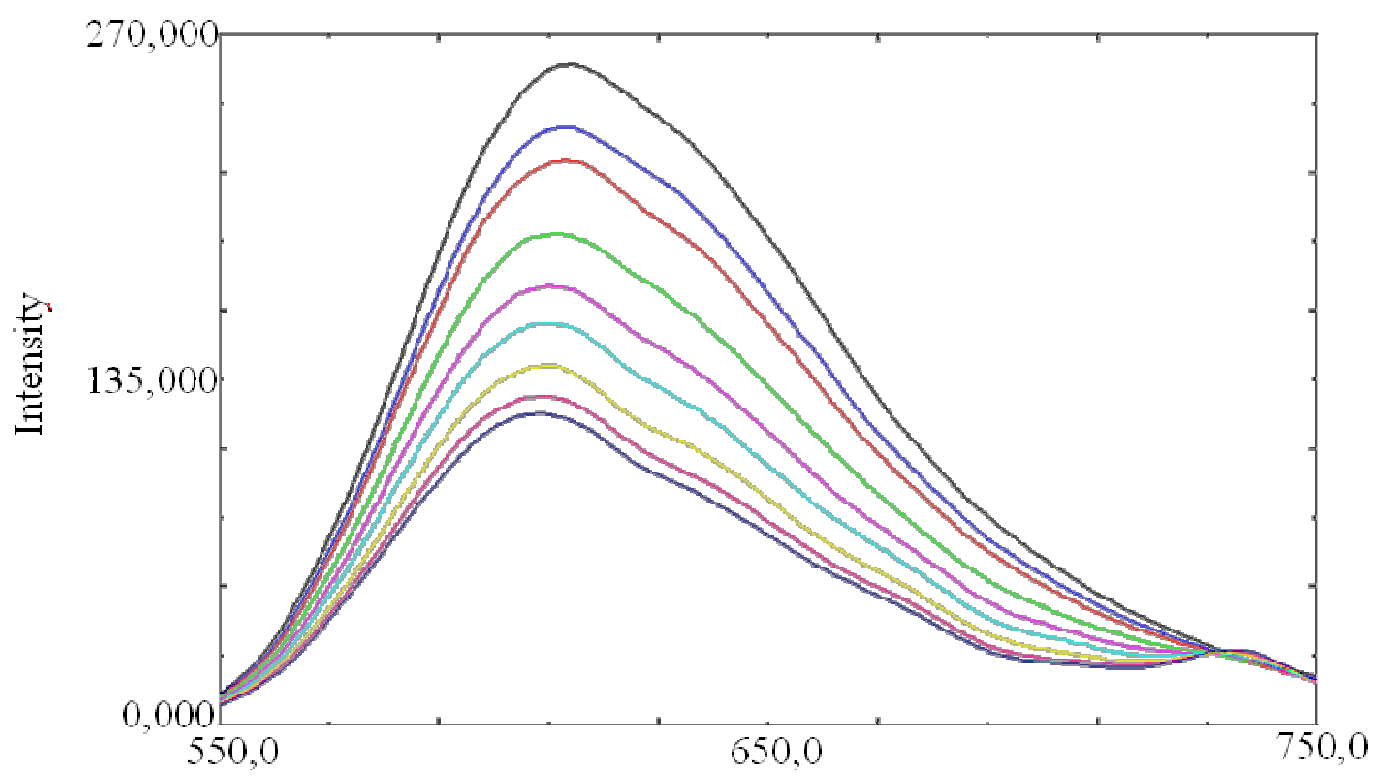

Wavelength (nm)

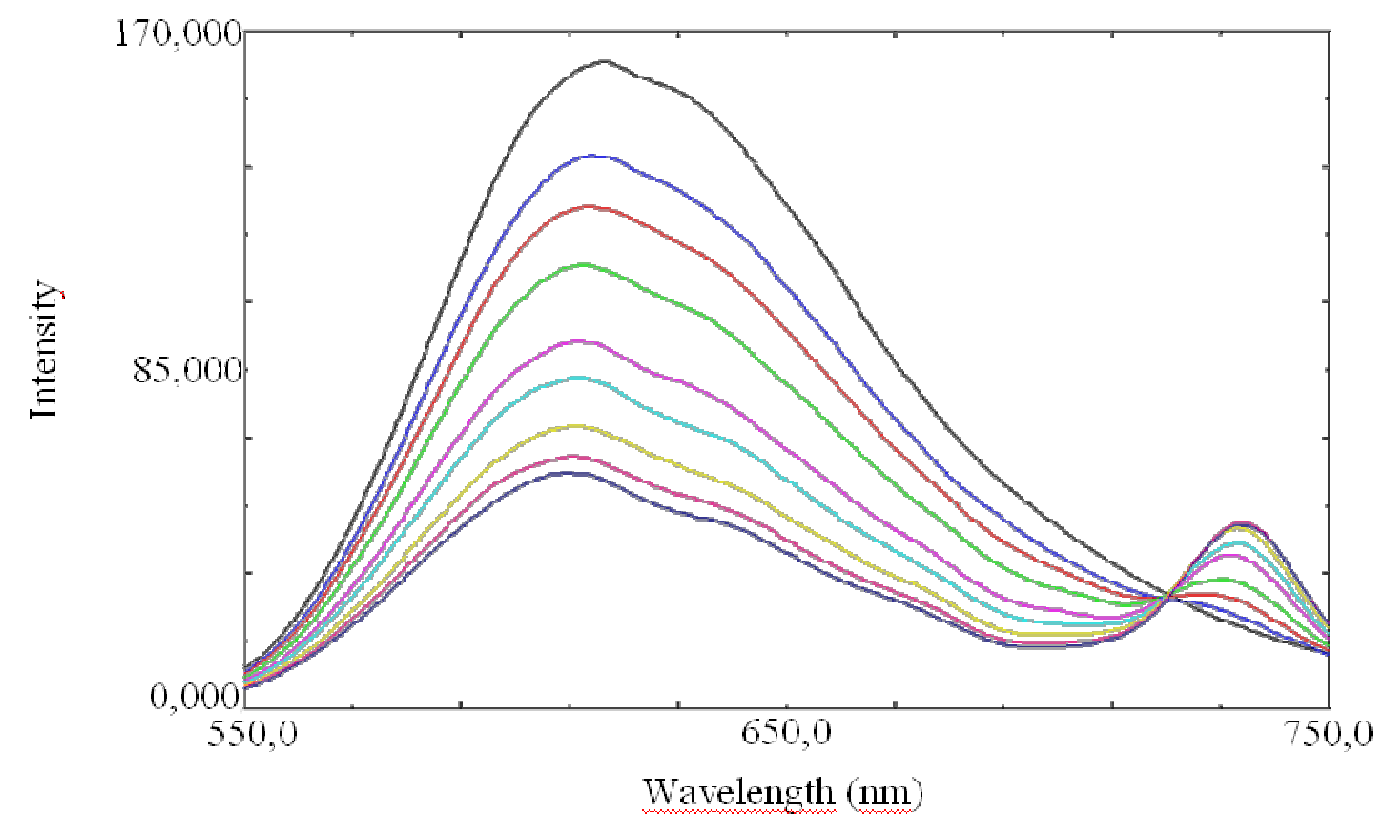

Figure 3b: Change in fluorometric intensity of $\mathrm{EtBr}-\mathrm{Tel} 21$ with addition of BtPc in the absence (up) and presence (down) of $150 \mathrm{mM} \mathrm{KCl}$. 


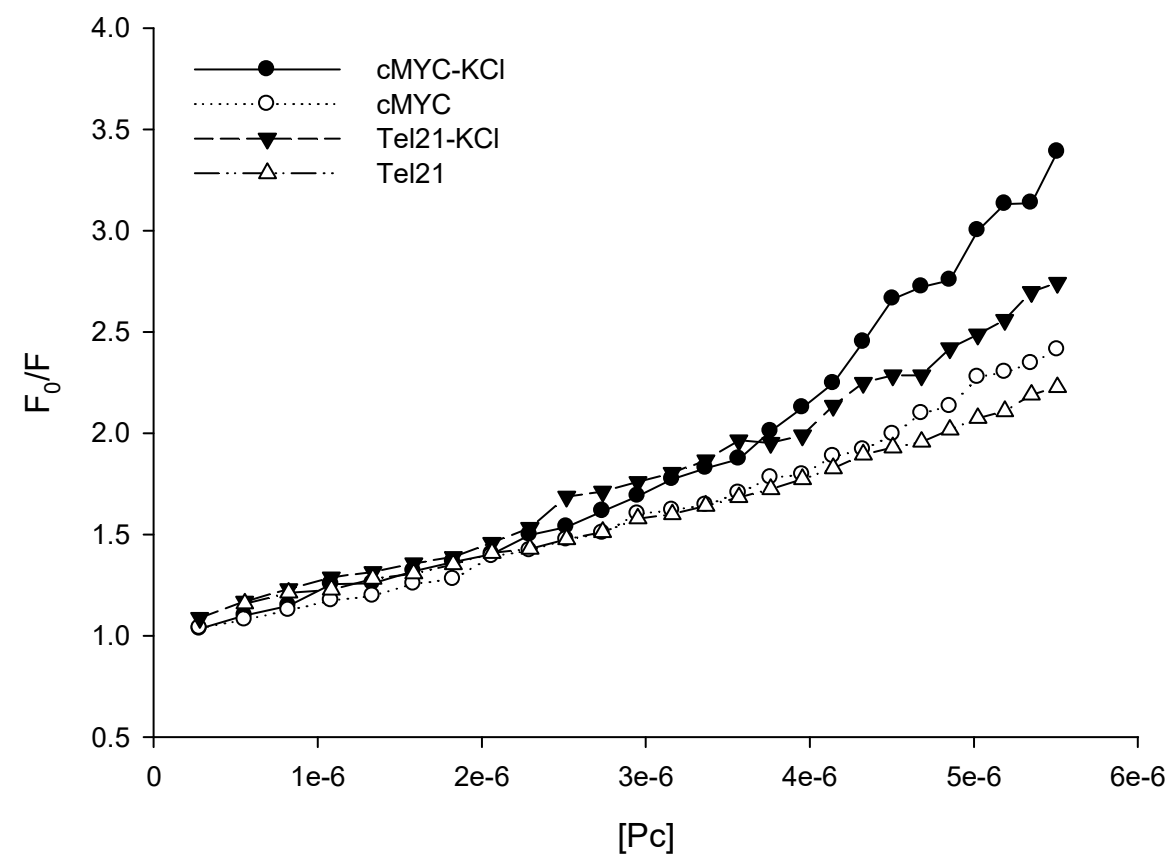

Figure 4: Stern-Volmer plots for quenching of EtBr-oligomer with addition of BtPc, regression coefficients $(r 2)$ are higher than 0.97 except cMYC in the presence of $\mathrm{KCl}$ (0.92).

Table 2: Stern-Volmer equations for $5.56 \times 10^{-7}-3.76 \times 10^{-6} \mathrm{M}$ of BtPc.

\begin{tabular}{llll}
\hline & & Stern-Volmer plot, $\mathrm{M}^{-1}$ & $\mathrm{r}^{2}$ \\
\hline \multirow{2}{*}{ Without KCl } & CMYC & $\mathrm{y}=2.2 \times 10^{5} \mathrm{x}+0.93$ & 0.99 \\
\multirow{2}{*}{ With KCl } & Tel 21 & $\mathrm{y}=1.8 \times 10^{5} \mathrm{x}+1.0$ & 0.99 \\
& CMYC & $\mathrm{y}=2.7 \times 10^{5} \mathrm{x}+0.91$ & 0.98 \\
& Tel 21 & $\mathrm{y}=2.6 \times 10^{5} \mathrm{x}+0.98$ & 0.98 \\
\hline
\end{tabular}

\section{CONCLUSION}

In our previous study, it was documented that BtPc has a potential usage for anticancer treatment due to its affinity to dsDNA. The certain sequences, like guanine rich segment of DNA, have the ability to form G-quadruplex structures. As the stabilization of G-quadruplex structure thought to be a promising target for cancer treatment, the binding and stabilization effect of BtPc was investigated in the present study. A telomeric and a proto-oncogenic sequence were investigated for the mentioned purposes. 
The highest binding constant was found for Tel 21 in the presence of $\mathrm{KCl}$, which indicated the stabilization effects of BtPc to mentioned G-quadruplex. The CD spectrums were also demonstrated the stabilization of both parallel and anti-parallel Tel $21 \mathrm{G}$-quadruplex in the presence of $\mathrm{KCl}$. Besides, the stabilization of anti-parallel form of G-quadruplex was achieved for Tel 21 in the absence of $\mathrm{KCl}$. The stability of the parallel structure was achieved for $\mathrm{CMYC}$ in the absence of $\mathrm{KCl}$ up to $4.10 \mu \mathrm{M}$ of Pc. The disruption of staking of parallel form was achieved for CMYC in the presence of $\mathrm{KCl}$. According to fluorometric data, in the presence of $\mathrm{KCl}$, the Stern-Volmer constants have the higher values for both Tel 21 and CMYC compared to values obtained in the absence of $\mathrm{KCl}$.

With the results achieved in the present study, binding of BtPc with G-quadruplex can be employed as a guide for new studies in the following ways: (i) As the Gquadruplexes are generally over expressed in cancer cells, BtPc molecules have the accumulation potential within cancer cells. (ii) G-quadruplexes are used as drug delivery vehicles; targeted delivery of BtPc to a certain cell/tumor is possible via conjugation with a specific G-quadruplex.

Conflict of interest: The authors declare that they have no conflict of interest.

\section{REFERENCES}

1 A. Ali, S. Bhattacharya, DNA binders in clinical trials and chemotherapy, Bioorganic Med. Chem. 22 (2014) 4506-4521.

2 M. Di Antonio, R. Rodriguez, S. Balasubramanian, Experimental approaches to identify cellular G-quadruplex structures and functions, Methods. 57 (2012) 84-92.

3 H. Han, L.H. Hurley, G-quadruplex DNA: A potential target for anti-cancer drug design, Trends Pharmacol. Sci. 21 (2000) 136-142.

4 A.J. Bhattacharjee, K. Ahluwalia, S. Taylor, O. Jin, J.M. Nicoludis, R. Buscaglia, et al., Induction of G-quadruplex DNA structure by Zn(II) 5,10,15,20-tetrakis(N-methyl-4pyridyl)porphyrin, Biochimie. 93 (2011) 1297-1309.

5 T.A. Brooks, L.H. Hurley, Targeting MYC Expression through G-Quadruplexes, Genes Cancer. 1 (2010) 641-649.

6 D.S.H. Chan, H. Yang, M.H.T. Kwan, Z. Cheng, P. Lee, L.P. Bai, et al., Structurebased optimization of FDA-approved drug methylene blue as a c-myc G-quadruplex DNA stabilizer, Biochimie. 93 (2011) 1055-1064.

$7 \quad$ V. Dapic, Biophysical and biological properties of quadruplex oligodeoxyribonucleotides, Nucleic Acids Res. 31 (2003) 2097-2107.

8 J. Moon, J.H. Han, D.Y. Kim, M. Jung, S.K. Kim, Effects of deficient of the Hoogsteen 
base-pairs on the G-quadruplex stabilization and binding mode of a cationic porphyrin, Biochem. Biophys. Reports. 2 (2015) 1-7.

9 E.W. White, Selective Recognition of Quadruplex DNA by Small Molecules by, (2006).

10 T. Simonsson, G-quadruplex DNA structures - Variations on a theme, Biol. Chem. 382 (2001) 621-628.

11 C. Gao, W. Zhang, S. He, S. Li, F. Liu, Y. Jiang, Synthesis and antiproliferative activity of 2,7-diamino 10 -(3,5-dimethoxy)benzyl-9(10H)-acridone derivatives as potent telomeric G-quadruplex DNA ligands, Bioorg. Chem. 60 (2015) 30-36.

12 A. De Cian, L. Lacroix, C. Douarre, N. Temime-Smaali, C. Trentesaux, J.-F. Riou, et al., Targeting telomeres and telomerase, Biochimie. 90 (2008) 131-155.

13 L. Zhang, J. Huang, L. Ren, M. Bai, L. Wu, B. Zhai, et al., Synthesis and evaluation of cationic phthalocyanine derivatives as potential inhibitors of telomerase, Bioorganic Med. Chem. 16 (2008) 303-312.

14 P. Charoenphol, H. Bermudez, Design and application of multifunctional DNA nanocarriers for therapeutic delivery, Acta Biomater. 10 (2014) 1683-1691.

15 E. Yabaş, E. Bağda, E. Bağda, The water soluble ball-type phthalocyanine as new potential anticancer drugs, Dye. Pigment. 120 (2015) 220-227.

16 G. De Torre, G. Bottari, U. Hahn, T. Torres, Functional Phthalocyanine Molecular Materials, 2010.

17 A.Y. Tolbin, A. V. Ivanov, L.G. Tomilova, N.S. Zefirov, Preparation of 1,2-bis(3,4dicyanophenoxymethyl)benzene and the binuclear zinc phthalocyanine derived from it, Mendeleev Commun. 12 (2002) 96-97.

18 L. Hassani, F. Hakimian, E. Safaei, Spectroscopic investigation on the interaction of copper porphyrazines and phthalocyanine with human telomeric G-quadruplex DNA, Biophys. Chem. 187-188 (2014) 7-13.

19 A.A. Salem, I.A. El Haty, I.M. Abdou, Y. Mu, Interaction of human telomeric Gquadruplex DNA with thymoquinone: A possible mechanism for thymoquinone anticancer effect., Biochim. Biophys. Acta. 1850 (2015) 329-42.

20 H. Yaku, T. Murashima, H. Tateishi-Karimata, S.I. Nakano, D. Miyoshi, N. Sugimoto, Study on effects of molecular crowding on G-quadruplex-ligand binding and ligand-mediated telomerase inhibition, Methods. 64 (2013) 19-27.

21 V. Kumar, A. Sengupta, K. Gavvala, R.K. Koninti, P. Hazra, Spectroscopic and thermodynamic insights into the interaction between proflavine and human telomeric Gquadruplex DNA., J. Phys. Chem. B. 118 (2014) 11090-9.

22 S. Neidle, M. a Read, G-quadruplexes as therapeutic targets., Biopolymers. 56 (2001) 195-208.

23 S. Neidle, Genomic Quadruplexes as Therapeutic Targets, Ther. Appl. Quadruplex Nucleic Acids. (2012) 119-138.

24 D. Ren iuk, I. Kejnovska, P. kolakova, K. Bedna ova, J. Motlova, M. Vorli kova, Arrangements of human telomere DNA quadruplex in physiologically relevant $\mathrm{K}+$ solutions, Nucleic Acids Res. 37 (2009) 6625-6634.

25 H. a Benesi, J.H. Hildebrand, A spectrophotometric investigation of the interaction of iodine with aromatic hydrocarbons, J. Am. Chem. Soc. 71 (1949) 2703-2707. 
26 H. Dezhampanah, T. Darvishzad, M. Aghazadeh, Thermodynamic and spectroscopic study on the binding of interaction anionic phthalocyanine with calf thymus DNA, Spectrosc. An Int. J. 26 (2011) 357-365.

27 J. Jaumot, R. Gargallo, Experimental Methods for Studying the Interactions between G-Quadruplex Structures and Ligands, Curr. Pharm. Des. 18 (2012) 1900-1916.

28 M. Sirajuddin, S. Ali, A. Badshah, Journal of Photochemistry and Photobiology B : Biology Drug - DNA interactions and their study by UV - Visible, fluorescence spectroscopies and cyclic voltametry, J. Photochem. Photobiol. B Biol. 124 (2013) 1-19.

29 S. Paramasivan, I. Rujan, P.H. Bolton, Circular dichroism of quadruplex DNAs: Applications to structure, cation effects and ligand binding, Methods. 43 (2007) 324-331.

30 M. Islam, S. Fujii, S. Sato, T. Okauchi, S. Takenaka, A Selective G-Quadruplex DNAStabilizing Ligand Based on a Cyclic Naphthalene Diimide Derivative, Molecules. 20 (2015) 10963-10979.

31 Q. Guo, M. Lu, L. a Marky, N.R. Kallenbach, Interaction of the dye ethidium bromide with DNA containing guanine repeats., Biochemistry. 31 (1992) 2451-2455.

32 F. Koeppel, J.F. Riou, a Laoui, P. Mailliet, P.B. Arimondo, D. Labit, et al., Ethidium derivatives bind to G-quartets, inhibit telomerase and act as fluorescent probes for quadruplexes., Nucleic Acids Res. 29 (2001) 1087-1096. 
